\title{
Methodologic Progress Note: Opportunistic Sampling for Pharmacology Studies in Hospitalized Children
}

\author{
Sonya Tang Girdwood, MD, PhD',2*, Jennifer Kaplan, MD, MS $3{ }^{3,4}$, Alexander A Vinks, PharmD, PhD²,
}

'Division of Hospital Medicine, Cincinnati Children's Hospital Medical Center, Cincinnati, Ohio; ${ }^{2}$ Division of Clinical Pharmacology, Cincinnati Children's Hospital Medical Center, Cincinnati, Ohio; ${ }^{3}$ Division of Critical Care Medicine, Cincinnati Children's Hospital Medical Center, Cincinnati, Ohio; ${ }^{4}$ Department of Pediatrics, University of Cincinnati, College of Medicine, Cincinnati, Ohio.

hallenges in conducting and completing studies of drugs in vulnerable populations, such as hospitalized children, include weak study designs and lack of sufficient sample sizes to achieve adequate power. ${ }^{1} \mathrm{Lim}$ itations in the amount of blood that can be safely drawn in children and low parental consent rates due to concerns for anemia or pain, if venipuncture is required, lead to an insufficient number of patients enrolled in traditional clinical studies. ${ }^{2}$ Thus, sample size targets are often not met. Recognizing the limited pediatric data for many drugs routinely prescribed off label in children, the Food and Drug Administration implemented the Best Pharmaceuticals for Children Act and the Pediatric Research Equity Act (PREA) in 2003; these legislative acts require clinical studies to assess the safety and efficacy of new drugs in children. ${ }^{1}$ While studies conducted under these acts have provided important information for the clinical care of children, only one-third of mandatory pediatric postmarketing studies of the 114 new drugs and new indications subject to PREA requirements between 2007 and 2014 had been completed within 7 years. ${ }^{3}$

Despite the challenges in conducting studies of drugs in children, robust pediatric data must be generated in children, especially in those with medical complexity or with chronic medical diseases and who have significant risk of experiencing adverse drug events. Data in adults cannot simply be extrapolated to children. ${ }^{4}$ In addition, studies from healthy children may not apply to hospitalized pediatric patients because of significant physiologic changes that occur in children who are ill enough to be hospitalized. Opportunistic sampling can provide robust drug disposition data and overcome some of the challenges encountered by traditional drug studies. In this Methodologic Progress Note, we describe the utility of opportunistic sampling as a research tool for hospitalists, in partnership with clinical pharmacologists, to study drug pharmacokinetics (PK) in hospitalized children.

\section{OPPORTUNISTIC SAMPLING DEFINED}

Opportunistic sampling relies on the use of blood samples that are ordered for clinical purposes, and its use is endorsed by the

*Address correspondence to: Sonya Tang Girdwood, MD, PhD; E-mail: Sonya. Tanggirdwood@cchmc.org; Telephone: 513-803-2690; Twitter: @STangGirdwood.

Published online first February 19, 2020.

Received: October 28, 2019; Revised: December 20, 2019;

Accepted: December 26, 2019

๑ 2021 Society of Hospital Medicine DOI 10.12788/jhm.3380
Pediatric Trials Network. ${ }^{2,5}$ Opportunistic sampling approaches have two types: sparse sampling and scavenged or remnant sampling. In sparse opportunistic sampling, additional blood is obtained at the same time clinical samples are ordered, which avoids the need for additional punctures. ${ }^{5}$ This approach requires bedside personnel to obtain additional blood that is sent to the research team for further processing. Scavenged sampling relies on leftover residual blood from clinical samples. ${ }^{2.5}$ After the clinical laboratory performs the laboratory test ordered by the clinical team, the research team can scavenge residual blood for measurement of drug concentrations. When drug concentrations are measured from multiple patients, clinical pharmacologists can perform population PK modeling to characterize the pharmacokinetics of the drug and its variability within the population level.

The Figure shows how scavenged samples from hypothetical patients could be used to generate a PK curve. In this example, three patients are admitted for osteomyelitis and treated with the same antibiotic administered every 8 hours, as shown by the theoretical concentration vs time profiles in the top panel. To determine the effectiveness of treatment and timing of transition to enteral antibiotics, the clinical team orders C-reactive protein (CRP) evaluation approximately every 48 hours for each patient. Patients have their first dose of antibiotic at a different time of day depending on the time of admission or surgical drainage. Therefore, the timing of the blood draw with respect to the most recent antibiotic dose varies between patients even if blood draws are ordered at the same time for all patients (ie, 4 Am lab draw). For patient 1, the blood draws occur approximately 3 to 5 hours after the second and eighth antibiotic doses (Figure, top panel). After the clinical laboratory measures the CRP, the research team scavenges residual blood from the lab samples and measures antibiotic concentrations. The antibiotic concentrations are then plotted on a concentration vs time after most recent dose. (Figure, bottom panel). Patient 2 has blood drawn 5 to 7 hours after the first and seventh antibiotic doses. The third patient has blood drawn within 2 hours after the second and eighth antibiotic doses. As more samples are collected from additional patients, population PK modeling provides a robust description of the central tendency of the concentration vs time profile. More important, as more patients are included, the interpatient variability in concentrations can be described within the population, which often cannot be performed well with the smaller numbers of patients enrolled in traditional PK studies.

Opportunistic sampling has advantages over traditional intensive PK studies that often require multiple blood draws 


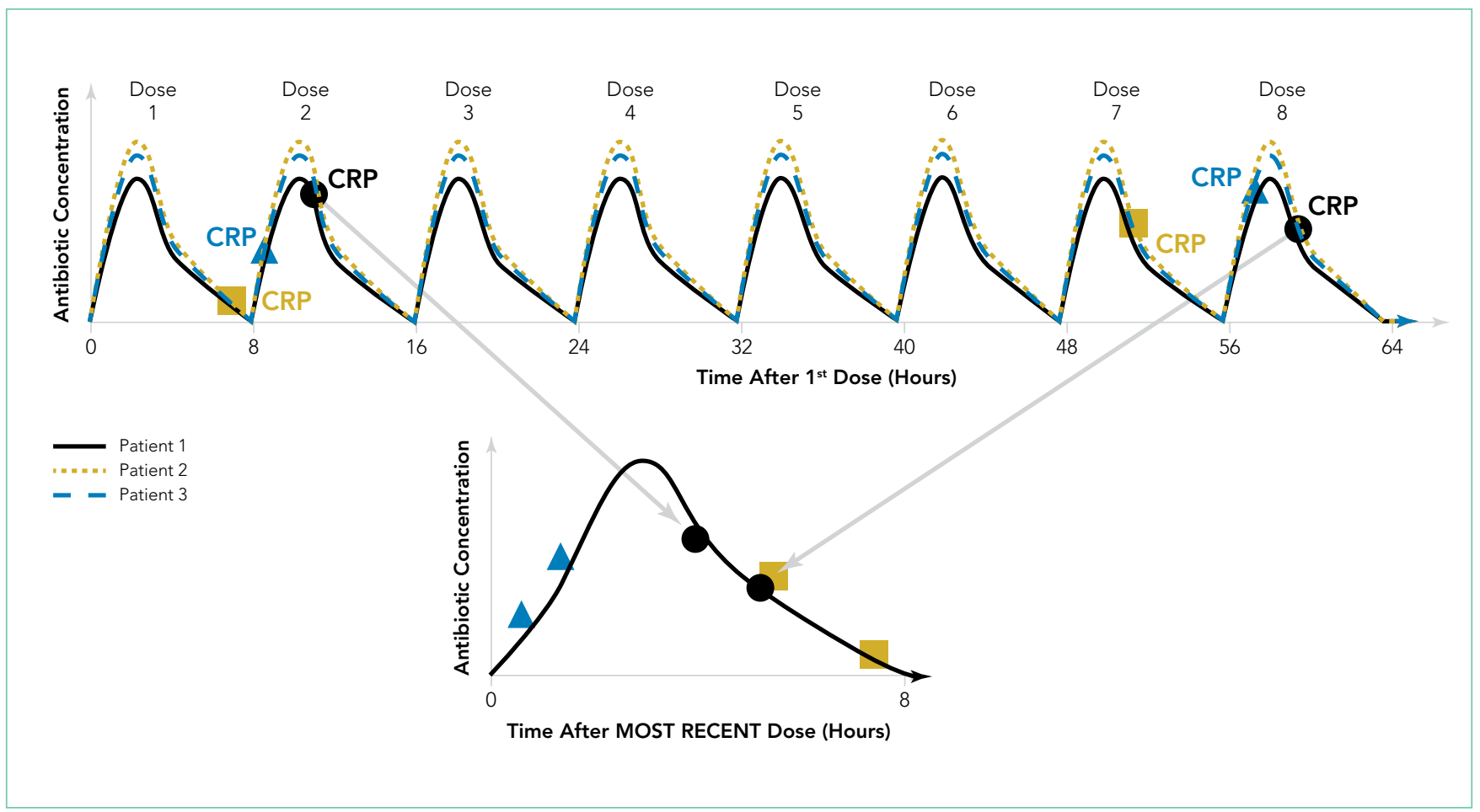

FIG. Illustration of how samples obtained from multiple hypothetical patients through opportunistic sampling can describe the concentration vs time profile at the population level. In this example, all three patients receive the same antibiotic every 8 hours, as shown by the concentration vs time profiles in the top panel (solid black, dotted yellow, and dashed blue lines). Each patient has two C-reactive protein (CRP) levels obtained approximately every 48 hours, as shown in the top panel. Patient 1 (black solid line) has blood drawn after the second and eighth doses for CRP measurement. Residual blood after CRP testing is used to measure the antibiotic concentration. The antibiotic concentrations measured from the two CRP samples from patient 1 are plotted on a concentration vs time after most recent dose graph, as shown in the bottom panel. Patient 2 (dotted yellow line) has blood drawn after the first and seventh doses. Patient 3 (dashed blue line) has blood drawn shortly after the second and eighth doses are given. Antibiotic concentrations measured from all three patients are plotted on the same concentration vs time after most recent dose profile to describe antibiotic pharmacokinetics at the population level, as shown in the bottom panel.

(typically >8-10) within one dosing interval to adequately describe the phases of absorption, distribution, metabolism, and elimination. The number of vascular punctures can be painful if blood cannot be drawn from existing vascular access, and the large amount of blood (sometimes $>1 \mathrm{~mL}$ per sample) required for these studies can be impractical, burdensome, and even dangerous in young children and neonates. Scavenged sampling reduces the risk of anemia because no additional blood is drawn beyond what is obtained for clinical purposes, and it does not disrupt nursing workflow or add to nursing workload. Approval to use scavenged blood requires approval from the institutional review board. At some institutions, the consent to treat form on admission may address the use of scavenged samples and therefore allow for waiver of consent. In addition, the consent process may occur retroactively after samples are collected. These methods lead to increased enrollment.

Limitations in this approach are that drugs may degrade over time in whole blood or processed samples. Therefore, the process by which the clinical laboratory stores the residual blood after clinical tests must be understood, and the stability of the drug or metabolite of interest in blood or plasma over time must be ensured. In addition, residual blood may not be present after a clinical test, and recording times of the drug administration and lab draws may be inaccurate..$^{2,5}$

\section{APPLICATIONS OF OPPORTUNISTIC SAMPLING IN CLINICAL PHARMACOLOGY RESEARCH}

Opportunistic sampling has been successfully used to study a variety of drugs in different pediatric populations but has been primarily used in neonates. The multicenter Pharmacokinetics of Understudied Drugs Administered to Children Per Standard of Care trial has utilized this approach to evaluate the PK of over 30 drugs. ${ }^{5}$ Several antimicrobials have been studied through opportunistic sampling, including those frequently used in pediatric hospital medicine, such as ampicillin ${ }^{6}$ and clindamycin. ${ }^{7}$

This sampling approach may be most beneficial in studying select patients. Patients who are obese, a group often excluded in pediatric drug trials, have been previously included in opportunistic drug studies. ${ }^{8}$ The utility of opportunistic sampling to study antimicrobials, morphine, and cardiac drugs has been demonstrated in neonates, both preterm and term, in whom additional blood draws can be challenging because of low total blood volume and limited vascular access. ${ }^{6,7,9-12}$

Although the frequency of blood draws from patients admitted to pediatric hospital medicine services is generally lower than that for patients on other subspecialty services, such as critical care, we can capitalize on the high volume of patients with common diagnoses (eg, pneumonia, skin, and soft tissue infections) who are admitted to hospital medicine. Using op- 
portunistic sampling, we can study the PK of drugs frequently used in hospital medicine, such as antibiotics, antiepileptic drugs, steroids, and pain medications. In addition, we can measure drug concentrations to study the effects of route administration (oral, enteric tube, or intravenous) to guide not only the dosing but also the timing of transition to enteral medications. Finally, we can study drugs that are commonly used in adult and pediatric patient populations cared for by hospitalists but who are often excluded from clinical drug trials, such as patients with medical complexity, patients with medical devices (eg, nervous system shunts and tracheostomies), patients taking concomitant medications, or patients on extracorporeal devices such as dialysis, to validate drug regimens.

\section{CONCLUSION}

Generating robust pediatric clinical pharmacology data has many inherent challenges because of the vulnerability of children. However, their vulnerability requires that medications be studied thoroughly in children to ensure their safety and effectiveness. Opportunistic sampling allows for rigorous studies to be conducted with adequate sample sizes while minimizing the risk of pain, anemia, and other adverse events related to clinical drug trials. Pediatric hospitalists should consider this approach to advance their knowledge of commonly used drugs that have not been adequately studied in hospitalized children and can expand the use of opportunistic sampling to study other aspects of disease, such as diagnostic or prognostic biomarkers.

Disclosures: The authors have no conflicts of interest relevant to this article to disclose.

Funding: Dr Tang Girdwood is supported by the National Institute of Child Health and Development Cincinnati Pediatric Clinical Pharmacology Postdoctoral Training Program (5T32HD069054-09), Cincinnati Children's Hospital Med- ical Center Arnold W Strauss Fellow Award, and Cincinnati Children's Hospital Medical Center Hospital Medicine Fellow Award.

\section{References}

1. Field MJ, Boat TF, eds. Safe and Effective Medicines for Children: Pediatric Studies Conducted Under the Best Pharmaceuticals for Children Act and the Pediatric Research Equity Act. National Academies Press; 2012

2. Laughon MM, Benjamin DK Jr, Capparelli EV, et al. Innovative clinical trial design for pediatric therapeutics. Expert Rev Clin Pharmacol. 2011;4(5):643652. https://doi.org/10.1586/ecp.11.43

3. Hwang TJ, Orenstein L, Kesselheim AS, Bourgeois FT. Completion rate and reporting of mandatory pediatric postmarketing studies under the US Pediatric Research Equity Act. JAMA Pediatr. 2018;173(1):68-74. https://doi. org/10.1001/jamapediatrics.2018.3416

4. Rieder M. Adverse drug reactions in children: pediatric pharmacy and drug safety. J Pediatr Pharmacol Ther. 2019;24(1):4-9. https://doi. org/10.5863/1551-6776-24.1.4

5. Balevic SJ, Cohen-Wolkowiez M. Innovative study designs optimizing clinical pharmacology research in infants and children. J Clin Pharmacol. 2018;58(10):S58-S72. https://doi.org/10.1002/jcph.1053

6. Tremoulet A, Le J, Poindexter B, et al. Characterization of the population pharmacokinetics of ampicillin in neonates using an opportunistic study design. Antimicrob Agents Chemother. 2014;58(6):3013-3020. https://doi. org/10.1128/AAC.02374-13

7. Gonzalez D, Melloni C, Yogev R, et al. Use of opportunistic clinical data and a population pharmacokinetic model to support dosing of clindamycin for premature infants to adolescents. Clin Pharmacol Ther. 2014;96(4):429-437. https://doi.org/10.1038/clpt.2014.134

8. Smith MJ, Gonzalez D, Goldman JL, et al. Pharmacokinetics of clindamycin in obese and nonobese children. Antimicrob Agents Chemother. 2017;61(4): e02014-16. https://doi.org/10.1128/AAC.02014-16

9. Leroux S, Turner MA, Guellec CB, et al. Pharmacokinetic studies in neonates: the utility of an opportunistic sampling design. Clin Pharmacokinet. 2015;54(12):1273-1285. https://doi.org/10.1007/s40262-015-0291-1

10. Dallefeld SH, Atz AM, Yogev R, et al. A pharmacokinetic model for amiodarone in infants developed from an opportunistic sampling trial and published literature data. J Pharmacokinet Pharmacodyn. 2018;45(3):419430. https://doi.org/10.1007/s10928-018-9576-y

11. Thakkar N, Gonzalez D, Cohen-Wolkowiez M, et al. An opportunistic study evaluating pharmacokinetics of sildenafil for the treatment of pulmonary hypertension in infants. J Perinatol. 2016;36(9):744-747. https://doi.org/10.1038/ jp.2016.79

12. Euteneuer JC, Mizuno T, Fukuda T, Zhao J, Setchell KD, Vinks AA. Large variability in morphine concentrations in critically ill neonates receiving standard of care postoperative pain-management. Clin Pharmacol Ther. 2018;103:S45-S45 